\title{
Parental Concerns about the Health of Adolescents with Intellectual Disability: A Brief Report
}

\author{
Madonna Tucker, Miriam Taylor Gomez, Therese Rey-Conde, and Nicholas Lennox
}

Queensland Centre for Intellectual and Developmental Disability, The University of Queensland, Mater Hospital, South Brisbane, QLD, 4101, Australia

Correspondence should be addressed to Miriam Taylor Gomez, m.taylor3@uq.edu.au

Received 1 December 2010; Accepted 11 April 2011

Academic Editor: Margaret Kyrkou

Copyright ( $\odot 2011$ Madonna Tucker et al. This is an open access article distributed under the Creative Commons Attribution License, which permits unrestricted use, distribution, and reproduction in any medium, provided the original work is properly cited.

Background. Parents of adolescents with intellectual disability are concerned about the future health and well-being needs of their children. Method. Qualitative data was collected as part of a cross-sectional descriptive study and semi-structured interviews were conducted with 32 parents. The results were themed. Results. Most parents discussed areas of their children's health which made them anxious about the future. These concerns were collated into five themes. Conclusion. The health and well-being themes were dependency, general health, challenging behaviours, and increasing support needs.

\section{Introduction}

Compared with the general population, adults with intellectual disability experience significant healthcare inequalities including general health screening, mental health support, women's health screening, and oral healthcare services [1], and this equally applies to adolescents with intellectual disability [2,3]. The majority of Australian adolescents (1119 years) with intellectual disability live with their parents who have the responsibility for the healthcare of their adolescent child $[4,5]$.

Parents are concerned about the health of their adolescents with intellectual disability [6-9] especially when they can no longer care for them [10]. As adolescents transition out of specialist-based pediatric care, they move to the primary care system and general medical practitioners (GPs); this creates additional parental concerns about their child's health $[11,12]$. GPs have expressed concern at being expected to take on a similarly intensive role [13]. This study states the major themes expressed by parents regarding the health of their adolescent with intellectual disability.

\section{Method}

During a cross-sectional descriptive study which examined the effect of health interventions, interviews with parents were undertaken. This was a six-month cross-sectional descriptive study with qualitative and quantitative data collected. Qualitative data was collected from adolescents with intellectual disability, parents, and the adolescents' teachers. The other findings are reported elsewhere [3]. In a semistructured interview parents were asked about the three main health issues relevant to their adolescent in the next ten years. The dominant themes are discussed here.

\section{Results}

Thirty-two parents participated, of which 31 were mothers, with a mean age of 46 years, with $21 \%$ having tertiary qualifications. They were employed in a range of occupations including home duties, teaching, small business, retail, and nursing. Their adolescent children were described through GP notes and parent/teacher reporting as having mild $(3 / 32)$, moderate (17/32), severe (11/32), and profound $(1 / 32)$ intellectual disability. Only $33 \%$ (11/32) said that their children were strong and healthy and they had no concerns for the future, and $58 \%(18 / 32)$ said that there were particular areas of their children's health that made them anxious about the future, those being dependency, general health, challenging behaviour, and increasing support needs. 
3.1. Theme One: Dependency. Parents made practical observations about the capacity of their children's independence in future health decisions: "He will always be very dependent on us, and the resources we provide and the professional advice we seek." A few parents spoke of independence and how the future was not such a worry for them. "Independence-he likes to have his own things. The (health) diary (the study intervention) is his way of telling people about his health without his mum having to talk all the time."

3.2. Theme Two: General Health. Weight was most frequently named as the biggest challenge. There were additional concerns about management of medication, epilepsy management, "staying healthy mentally," mobility reduction over time, "getting herself to and from the doctor's when she is not feeling well and knowing when to go," "moving her from the child health to adult health system," and maintenance of health checks.

3.3. Theme Three: Challenging Behaviours. Parents consider that others outside the school environment will not know about "behavioural issues related to his condition." Another added that her main concern was that of "anger management" and another because "his behavioural issues may affect his social and daily life."

3.4. Theme Four: Increasing Support Needs. Parents perceived that they will need support for their own health as they age: "Support structures-we will need support in place as he and I get older"; "Advocacy_someone else needs to be aware of his health needs"; "Someone else to know his normal health-related patterns would be helpful. As his primary care-provider I may not always be around."

\section{Conclusion}

These findings are not a comprehensive list of parental concerns, but they contribute to our common understanding of parental health concerns for the future of their adolescent with intellectual disability.

\section{Acknowledgment}

This project was funded by the Queensland Health.

\section{References}

[1] E. Emerson and S. Durvasula, "Health inequalities and people with intellectual disabilities: an introduction to the special issue," Applied Research in Intellectual Disabilities, vol. 18, pp. 95-96, 2005.

[2] S. J. Geenen, L. E. Powers, and W. Sells, "Understanding the role of health care providers during the transition of adolescents with disabilities and special health care needs," Journal of Adolescent Health, vol. 32, no. 3, pp. 225-233, 2003.

[3] N. Lennox, T. Rey Conde, and S. Faint, "A pilot of healthcare interventions for adolescents with intellectual disability," Journal of Applied Research in Intellectual Disabilities, vol. 21, no. 5, pp. 484-489, 2008.
[4] Australian Institute of Health \& Welfare, Disability Prevalence and Trends, Australian Institute of Health and Welfare, Canberra, ACT, Australia, 2003.

[5] X. Wen, The Definition and Prevalence of Intellectual Disability in Australia, Australian Institute of Health and Welfare, Canberra, ACT, Australia, 1997.

[6] L. Abbeduto, M. M. Seltzer, P. Shattuck, M. W. Krauss, G. Orsmond, and M. M. Murphy, "Psychological well-being and coping in mothers of youths with autism," American Journal on Mental Retardation, vol. 109, no. 3, pp. 237-254, 2004.

[7] G. Browne and P. Bramston, "Parental stress in families of young people with an intellectual disability: the nurses role," The Australian Journal of Advanced Nursing, vol. 15, no. 3, pp. 31-37, 1998.

[8] R. H. Moos, "Life stressors, social resources, and coping skills in youth: applications to adolescents with chronic disorders," Journal of Adolescent Health, vol. 30, no. 4, supplement 1, pp. 22-29, 2002.

[9] N. White and R. P. Hastings, "Social and professional support for parents of adolescents with severe intellectual disabilities," Journal of Applied Research in Intellectual Disabilities, vol. 17, no. 3, pp. 181-190, 2004.

[10] M. Henwood, "Home and away: carers' health and well-being in the UK and Australia-similarities and differences," in Proceedings of the Share the Care National Carers Conference, Brisbane, Australia, 2000.

[11] L. Fiorentino, D. Phillips, A. Walker, and D. Hall, "Leaving paediatrics: the experience of service transition for young disabled people and their family carers," Health and Social Care in the Community, vol. 6, no. 4, pp. 260-270, 1998.

[12] R. W. Blum, D. Garell, C. H. Hodgman et al., "Transition from child-centered to adult health-care systems for adolescents with chronic conditions: a position paper of the Society for Adolescent Medicine," Journal of Adolescent Health, vol. 14, no. 7, pp. 570-576, 1993.

[13] B. O'Connell, S. Bailey, and J. Pearee, "Straddling the pathway from paediatrician to mainstream health care: transition issues experienced in disability care," Australian Journal of Rural Health, vol. 11, no. 2, pp. 57-63, 2003. 


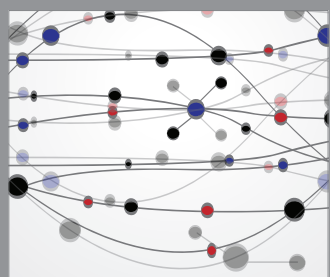

The Scientific World Journal
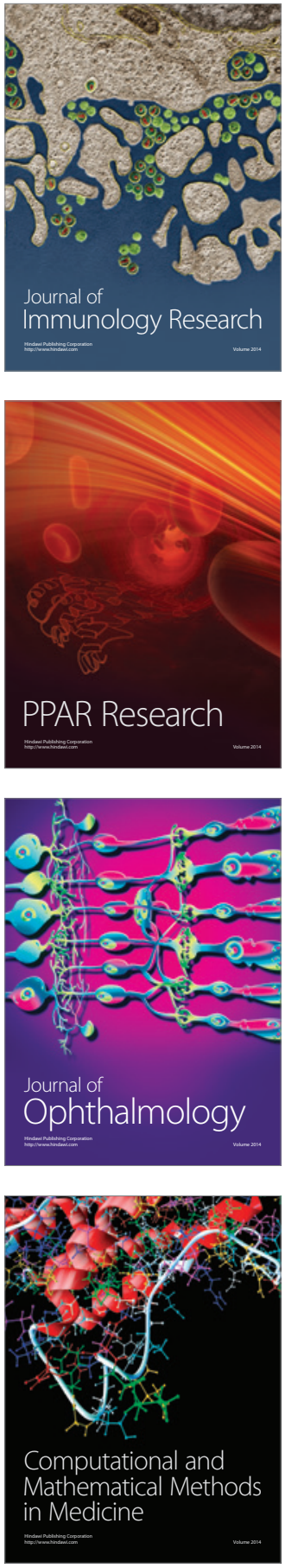

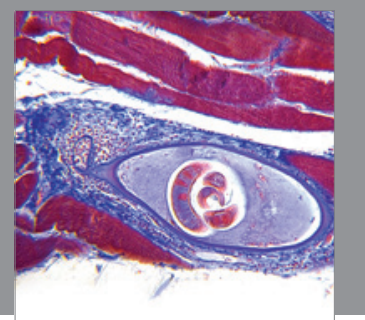

Gastroenterology

Research and Practice
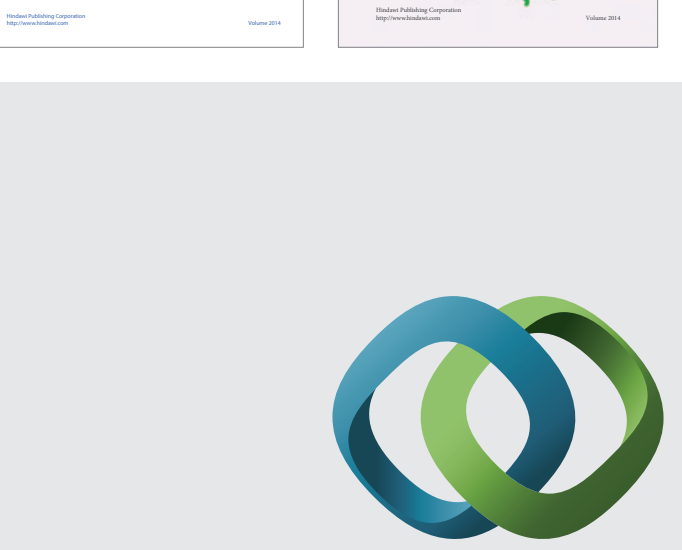

\section{Hindawi}

Submit your manuscripts at

http://www.hindawi.com
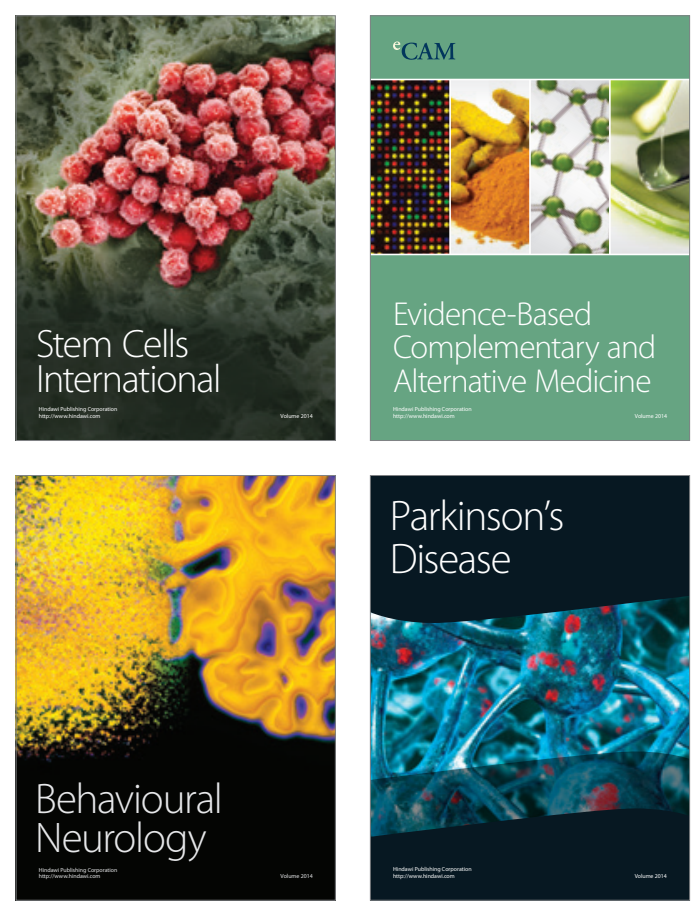

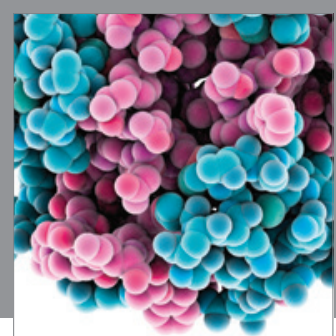

Journal of
Diabetes Research

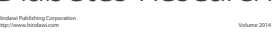

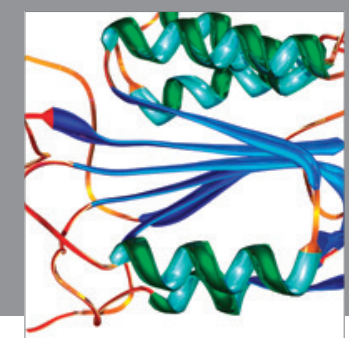

Disease Markers
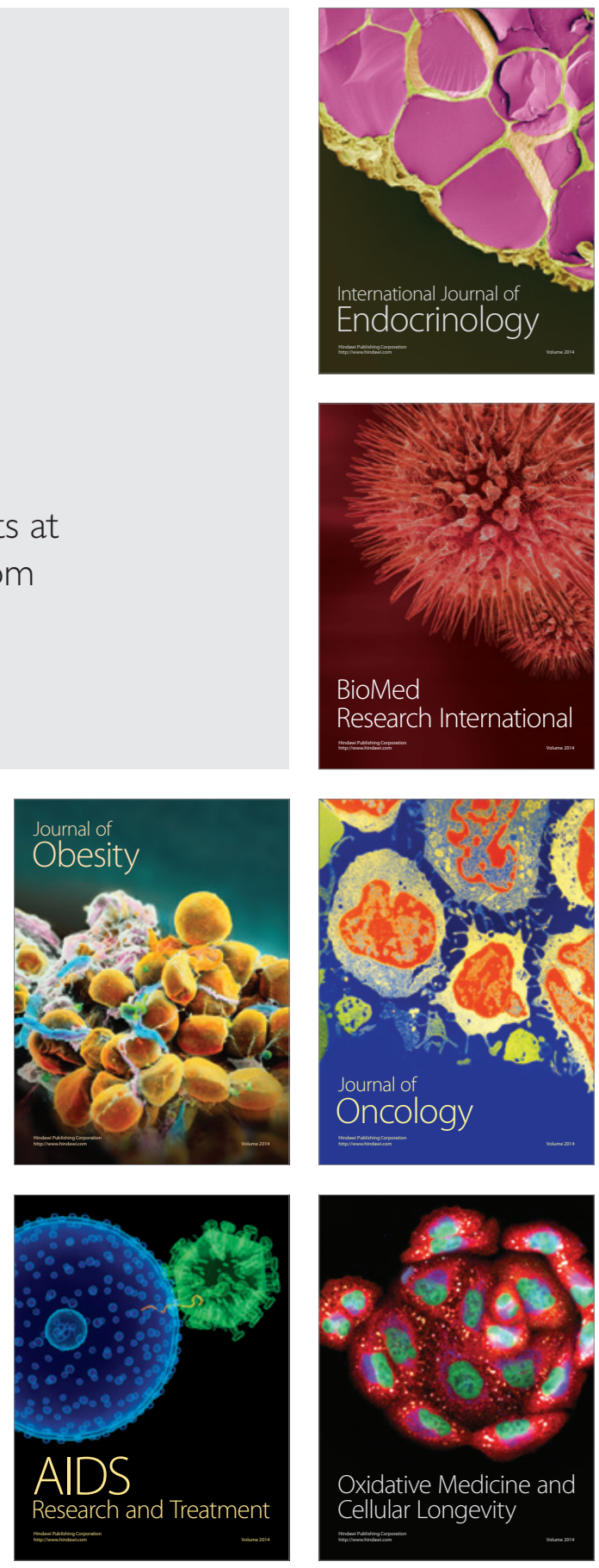